\title{
Development and delivery of pharmacy services for the London 2012 Olympic and Paralympic Games
}

\author{
Mark Stuart, ${ }_{1}^{1}$ David Mottram, ${ }^{2}$ David Erskine, ${ }^{3}$ Stephen Simbler, ${ }^{4}$ Trudy Thomas ${ }^{5}$
}

${ }^{1}$ International Forum on Quality and Safety in Health Care, BMJ Group, London, UK ${ }^{2} \mathrm{School}$ of Pharmacy and Biomolecular Sciences, Liverpool John Moores University, Liverpool, UK ${ }^{3}$ Pharmacy Department, Guy's and St Thomas' NHS Foundation Trust, London, UK ${ }^{4}$ MedsOnTrack Ltd, Harrow, UK ${ }^{5}$ Medway School of Pharmacy, Universities of Kent and Greenwich, Chatham Maritime, UK

Correspondence to Professor David Mottram, School of Pharmacy and Biomolecular Sciences, Liverpool John Moores University, Liverpool L3 3AF, UK; dr.mottram@btinternet.com

Received 30 October 2012 Revised 16 November 2012 Accepted 23 November 2012
To cite: Stuart $\mathrm{M}$, Mottram D, Erskine D, et al. Eur I Hosp Pharm 2013;20:42-45.

\section{ABSTRACT}

The London Organising Committee for the Olympic and Paralympic Games was established to plan and deliver the London 2012 Games. Within the Medical Services network, the Pharmacy Planning Committee (PPC) developed and delivered pharmacy services that were among the most advanced and comprehensive of any Games. The PPC designed a pharmacy service according to UK standards, operated through three polyclinics at the Athlete Villages. From these polyclinic pharmacies a system was set up for the supply, storage and management of medicines for use at all competition and training venues. To ensure safe and standardised approaches were used, a comprehensive set of policies and standard operating procedures was developed. Pharmacy guides, including drug formularies, were provided for the medical prescribers both within the polyclinics and as members of the Olympic and Paralympic Teams visiting the Games from around the world. Over 100 pharmacists and pharmacy technicians were recruited as the volunteer workforce for the Games. A training and education programme was developed to prepare this team to work in the unique environment of an Athlete Village polyclinic. A total of 5200 prescriptions (6849 items) were dispensed through the polyclinic pharmacies over the period of the Games. In addition, a Pharmacy Minor Ailments Scheme for nonathletes was introduced for the first time at an Olympics, with 1046 encounters taking place over the period of the Games. Valuable lessons were learnt from developing and delivering these pharmacy services. These have been reported back to the International Olympic Committee to ensure continued development and improvement of pharmacy services at future Games.

\section{INTRODUCTION}

The 2012 Olympic and Paralympic Games were awarded to the City of London in 2005. The London Organising Committee for the Olympic and Paralympic Games (LOCOG) was established to plan and deliver the Games. Within the LOCOG Medical Services network, the Pharmacy Planning Committee (PPC) began its role in 2008 under the leadership of Mark Stuart. The PPC members included the authors of this paper and Frances Akinwumni, Hayley File, Margaret Hagan and Simon Keady. Each member brought expertise and experience from across the sphere of pharmacy practice.

In the years prior to the Games the PPC met monthly at LOCOG's headquarters in London. Significant additional time was spent liaising with colleagues within LOCOG and with national and international pharmacy institutions and organisations.

\section{MEDICAL SERVICES FOR LONDON 2012}

LOCOG Medical Services comprised a number of work streams including pharmacy, sports medicine, primary care, physiotherapy, emergency medicine, dentistry, optometry, podiatry and medical imaging. These services operated through three polyclinics at the Athlete Villages at Stratford (Olympic Park), Eton Dorney (rowing and canoe sprint) and Weymouth (sailing). Over 4000 volunteer healthcare professionals were recruited by LOCOG to provide the medical services for the period of the Olympic and Paralympic Games.

Medical services were provided free of charge to 250000 LOCOG accredited personnel including athletes, their team officials, Olympic and Paralympic family members, technical officials and the LOCOG volunteer workforce. In addition, emergency medical support was provided for an estimated nine million spectators.

The period for which these services operated was from the opening of the Athlete Villages on 9 July to their closing after the Paralympic Games on 12 September. Polyclinics were open daily from 07:00 to 23:00, incorporating two shifts, with emergency cover outside these hours. In addition to services within the Athlete Villages, medical cover extended to 145 medical rooms located in all the competition and training venues.

\section{OVERVIEW OF PHARMACY SERVICES AT LONDON 2012}

Pharmacy services at the London 2012 Olympic and Paralympic Games were among the most advanced and comprehensive of any Olympic Games. The remit of the London 2012 pharmacy services was as follows:

- To design and oversee a safe, efficient and accurate dispensary service according to UK and Olympic and Paralympic medical standards

- To design a system of supply, storage and management of medicines for use at competition and training venues

- To facilitate the medicines licence arrangements of all Games pharmacy premises and venue facilities with UK pharmacy bodies including the Home Office, General Pharmaceutical Council and the Medicines and Healthcare Regulatory Agency (MHRA)

- To coordinate the registration and inspection of each of the pharmacy premises

- To develop and deliver a pharmacy training and education programme for the pharmacy workforce in collaboration with the national 
education organisation the Centre for Pharmacy Postgraduate Education (CPPE)

- To implement a bespoke standalone patient dispensing system for use at the Village pharmacies

- To deliver an international medicines information service to be available to all medical professionals during the Games

- To coordinate the writing, editing and review of the Olympic and Paralympic formularies

- To write the policies and procedures for all pharmacy-related activities that comply with the legal obligations of drug management in the UK

- To develop systems for dispensing and delivery of controlled drugs to individual patients and mobile doctors' kits for competition sites and spectator areasm

Details on these pharmacy services are presented below.

\section{POLYCLINIC PHARMACIES' FACILITIES AND RESOURCES}

The polyclinic pharmacies were designed to operate in a similar fashion to a hospital outpatient department. The environment within the polyclinics allowed for a close working relationship with the other volunteer healthcare professionals. In addition, the pharmacy staff enjoyed interaction with around 1000 medical practitioners from the 204 Olympic and 164 Paralympic countries that attended the Games. There was an interpreter system available, when necessary, to enable affective communication with athletes and their support staff.

A Games prescription pad was distributed through the polyclinic pharmacies and all medical encounters were recorded through an electronic medical encounter system.

\section{Pharmacy dispensing system}

A web-based pharmacy dispensing system was chosen as the pharmacy medical record system for the Games. The system helped coordinate the ordering of medicines for over 44 venues where drugs were kept. A multiple order pad system was available at the main Stratford pharmacy site that would allow a single point of ordering for every site. Orders for individual sites could be transmitted and the supplier would deliver the drugs to the specified site, usually within $24 \mathrm{~h}$.

All the drugs that were on the World Anti-Doping Agency (WADA) Prohibited List (http://www.wada-ama.org) were flagged in the system so that, should a drug that is on the WADA list be dispensed to a patient, a warning would appear and the pharmacist had to enter their password to confirm that the patient was aware of the drug status.

\section{PHARMACY GUIDE AND FORMULARY}

The aim of the London 2012 Olympic Games pharmacy guide was to provide comprehensive prescribing information on medicines that had been selected as being suitable for use during the Games and to describe the essential information needed by National Olympic Committee (NOC) healthcare personnel in relation to the use and supply of medicines.

Two years prior to the Games a medicines list was compiled by comparing the contents of previous Olympic formularies and identifying UK equivalent medicines. The focus was to provide a comprehensive set of medicines to cater for the specific requirements of sports medicine and that represented medicines for immediate treatment of patients presenting to the polyclinic for a range of likely conditions.

A consultation period for the medicines list was conducted prior to writing of the final pharmacy guide. The list was reviewed by the International Olympic Committee, International Paralympic Committee, National Pharmacy
Association, British National Formulary, a number of NHS hospital pharmacy departments, sports medicine experts and the members of the various LOCOG work streams.

The pharmacy guide included 178 items in the formulary. The drug monographs in the formulary were based on those published in the British National Formulary, but were edited to cater for the specific information needs in the Olympic and Paralympic environment. Each monograph in the book contained practical information for each drug on its indications, cautions, contraindications, available forms and side effects. The WADA status was also provided for each medicine in the formulary.

\section{POLICIES AND PROCEDURES RELATING TO PHARMACY SERVICES}

To comply with UK law and to ensure a safe and standardised approach by all pharmacy workforce, a comprehensive set of policies and standard operating procedures were developed in collaboration with national pharmacy organisations. The National Pharmacy Association provided close support in the development of these documents.

The standard operating procedures covered all aspects of pharmacy services including:

- Pharmacy management

- The storage and supply of medicines including Controlled Drugs

- Prescribing and dispensing

- Medicines information

- The Minor Ailments Scheme

Policies and procedures were sent to all health service personnel as appropriate in advance of the Games.

\section{DRUG SUPPLIES AND STOCK CONTROL TO VENUES}

The drug distribution systems to the three pharmacies and to the competition venues were designed so that one replicable model would apply to all venues, irrespective of the events taking place.

The aim was to provide for 'urgent and immediate' care for most anticipated medical incidents. If more complex or extended care was required, the patient would be referred to a designated hospital.

\section{PHARMACY VOLUNTEER WORKFORCE \\ Recruitment}

Recruitment of volunteers was undertaken by the LOCOG. One hundred pharmacy staff members were required to operate the three polyclinic pharmacies for the period of the Games. Games Maker advertisements were published in 2012 and expressions of interest were received from over 1200 pharmacists and pharmacy technicians.

The PPC drew up a short list of suitable applicants who were interviewed by the Games Maker interview panel. Around 100 volunteers were recruited. Volunteers agreed to commit to at least 10 working shifts during the Olympic and/or Paralympic Games.

\section{Management}

Rosters were designed for the pharmacy volunteers to ensure a balance of experience and expertise and guaranteeing that one or more senior member was included on duty for each shift. In April 2012, pharmacy volunteers were advised of their shift rosters which comprised an average of 10 shifts per volunteer. A substantial degree of management was required to 
accommodate the many requests for shift changes that arose due to non-Games work and social commitments of the volunteers.

\section{EDUCATION, TRAINING AND INFORMATION \\ Development plan}

From its first meeting, the PPC embarked on the development of a programme of education, training and information. This programme was produced in close collaboration with LOCOG Medical Services in order to produce education and training specifically designed for pharmacy volunteers but fully integrated with the generic training programme for all Medical Services volunteers.

It was also decided at an early stage to ensure that education and training for the Games would provide a legacy for the profession of pharmacy, both nationally and internationally.

\section{Education}

The provision of pharmacy services at the Games would require volunteers to have knowledge and skills in a variety of areas beyond normal professional duties, particularly with respect to the provision of medicines to athletes.

A major education programme was therefore undertaken in collaboration with the CPPE, a national education provider for pharmacy. A planning team was set up which included representatives from the Organising Committee and CPPE. The programme, which is delivered as an online e-learning programme, took 12 months to develop. It is entitled 'The Use of Drugs in Sport: A Healthcare Professional's Perspective' and includes three sections: (1) doping and anti-doping in sport; (2) pharmacy services and support in sport and fitness; and (3) medical services at international sporting events.

The primary objective of the programme was to provide a body of knowledge to support the role of pharmacist and pharmacy technician volunteers at the Games. The pharmacy volunteers were required to undertake the package and to pass the associated assessment as a prerequisite for acceptance as volunteers. The elearning package has also been made available to all pharmacists, pharmacy technicians and other healthcare professionals in the UK through the CPPE website (http://www.cppe.ac.uk).

In May 2012 the e-learning programme received the 'Get Set Award for Inspiring Outstanding Learning through the London 2012 Games', awarded by Podium, the Further and Higher Education Unit for the 2012 Games.

\section{Training}

Training for pharmacy Games Maker volunteers was designed and delivered in collaboration with the LOCOG Medical Services team. Training was in three phases. Orientation was delivered by LOCOG to all 70000 LOCOG volunteers. Role-specific training was designed by the pharmacy team and was repeated over seven training days, each of which included generic sessions for all Medical Services volunteers with a breakout session specifically designed for pharmacy volunteers. All pharmacy Games Makers were required to attend one of these days. Venue-specific training was delivered at the polyclinics within the three Athlete Villages and gave volunteers an opportunity to experience their Games-time work environment and the facilities that would be available to them within the pharmacy.

Prior to the Games, all pharmacy volunteers were given access to an online training programme for the dispensing system used at the polyclinic pharmacies. At the venue-specific training, pharmacists had a tutorial and hands-on training for this system.

A bespoke training programme was also developed for the pharmacist volunteers who were working in support of the athletes and accredited personnel at the football city competition venues.

\section{DRUG USAGE AT THE GAMES}

A total of 5200 prescriptions were dispensed over the whole opening period of the polyclinic pharmacies, including both the Olympic and Paralympic periods, with 6849 individual items dispensed over the whole Olympic and Paralympic operational period.

For the operational period of the Olympic Games, 3178 prescription forms were written for 4166 individual medicines orders. As in previous Games, the vast majority of items were for acute conditions and were included in the formulary. A breakdown of the most commonly prescribed items during the Olympic Games is shown in table 1.

Of the items prescribed, $92 \%$ were in the formulary. Of the 351 items prescribed that were not in the formulary, none were prescribed more than twice. The majority of non-formulary items prescribed were to continue chronic treatments (antihypertensives, cholesterol-lowering medicines, oral contraceptives, anti-epileptic agents, insulins), a few were for medicines where the clinician was unwilling to accept that the formularyequivalent agent was a suitable alternative as they were not familiar with it, and a few were for dressings and disinfectants (as there was no easily accessed alternative supply route available). Overall, the 178 medicines included in the formulary provided an adequate range of therapeutic options to meet the vast majority of clinical needs encountered in the medical centres. However, because of the need to also meet requirements to ensure continuity of ongoing treatments, the Games pharmacies also needed to ensure access to regular deliveries from a wholesaler.

A total of 2038 prescriptions were dispensed during the Paralympic Games. This was for a total of 2701 individual medicine items.

\section{PHARMACY MINOR AILMENTS SCHEME}

A Minor Ailments Scheme was developed with the aim of using the professional skills of the pharmacy volunteer workforce

Table 1 Most commonly prescribed items during the Olympic Games (prescribed more than 50 times)

\begin{tabular}{lll}
\hline Medicine & $\begin{array}{l}\text { No of } \\
\text { prescriptions }\end{array}$ & $\begin{array}{l}\text { Percentage of total } \\
\text { prescriptions dispensed }\end{array}$ \\
\hline Paracetamol 500-mg tablets & 456 & 10.95 \\
lbuprofen 400-mg tablets & 162 & 3.89 \\
Diclofenac sodium 50-mg & 132 & 3.17 \\
gastro-resistant tablets & & \\
lbuprofen 200-mg tablets & 96 & 2.30 \\
Strepsil lozenges & 96 & 2.30 \\
Amoxicillin 500-mg capsules & 87 & 2.09 \\
Hydrocortisone 1\% cream & 79 & 1.90 \\
Amoxicillin 250-mg capsules & 78 & 1.87 \\
Xylometazoline 0.1\% nasal spray & 77 & 1.85 \\
Cetirizine 10-mg tablets & 73 & 1.75 \\
Loratadine 10-mg tablets & 72 & 1.73 \\
Omeprazole 20-mg & 63 & 1.51 \\
gastro-resistant capsules & & \\
Diclofenac 1\% gel & 60 & 1.44 \\
Diclofenac sodium 75-mg & 57 & 1.37 \\
modified-release tablets & & \\
\hline
\end{tabular}


and, in so doing, relieving the workload on fellow healthcare professionals at the Games polyclinics.

The Minor Ailments Scheme only included over-the-counter medicines, which pharmacists could legally recommend as part of their current practice. No prescription-only medicines were included. The scheme was to be operated in the three Athlete Village polyclinic pharmacies (Stratford, Eton Dorney and Weymouth).

Pharmacists provided a professional consultation service for non-athletes presenting with one or more of a number of specific conditions. Olympic family members and workforce personnel were able to self-refer to the scheme or were referred by the polyclinic or other healthcare providers. In each case, the pharmacist undertook a private confidential consultation which consisted of:

- Assessment of the symptoms described

- Reference to the appropriate Minor Ailments Scheme protocol

- Provision of healthcare advice

- Provision of medication from the Minor Ailments Scheme formulary, if necessary and appropriate to the presenting condition.

All consultation details were entered on the LOCOG Electronic Record System.

If in the opinion of the pharmacist the person presented with symptoms outside the scheme, symptoms which indicated the need for urgent referral or a condition that had serious adverse implications for the health of the individual or others, the person was referred back to the polyclinic or other healthcare provider using the Minor Ailment Referral Form.

All pharmacy consultations, including details on the advice and medication given and referral where appropriate, were recorded on the person's Olympic medical record.

\section{Analysis of the Minor Ailments Scheme}

The Minor Ailments Scheme saw 1046 encounters over the period of the Games, with almost the same numbers of encounters during both the Olympic and Paralympic Games.

Workforce consultations accounted for approximately half of the encounters. The most commonly treated ailments were: headache (185 consultations), musculoskeletal conditions (126), general cold symptoms (109), sore throat (alone) (73), hay fever (65) and insect bites (54). Paracetamol was the medicine counter-prescribed most often (in 250 encounters), followed by ibuprofen (106 encounters).

\section{MEDICINES INFORMATION SERVICES FOR LOCOG STAFF AND TEAM DOCTORS}

Access to robust medicines information resources was required to ensure that clinic-based pharmacy staff could access safe and accurate information on both local and international medicines. In addition, the provision of a Medicines Information Service was required to support the local LOCOG and visiting team doctors in their practice in the UK medical environment.

It was decided that the internet-based 'Medicines Complete' package of information resources provided by the Pharmaceutical Press should be used as the first-line resource for enquiries that could not be immediately answered by pharmacy staff. In addition, Guy's and St Thomas' NHS Foundation Trust agreed to provide a $24 \mathrm{~h}$ on-call back-up service for the duration of the Games.

\section{Reported use of the medicines information service}

As highlighted above, it was felt that the pharmacy workforce should be empowered to answer as many enquiries themselves as possible before referring to the Medicines Information Service at Guy's and St Thomas' NHS Foundation Trust for additional support. Experience from these Games suggested that this was a very successful strategy. The following online reference resources were used by pharmacy staff to inform answers given to medicine information enquiries:

- British National Formulary (150 times, but all medical staff also had access to paper copies)

- Martindale online, the complete drug reference $(770$ times)

- Dietary Supplements (23 times)

- Kucers' The Use of Antibiotics (42 times)

On five occasions the pharmacy staff required additional support from the Medicines Information Service and on each occasion that service was able to answer the enquiry within an acceptable timeframe. The medicines information support service was not required out of hours but, given that the clinic opening hours were from 07:00 to 23:00, it would seem appropriate to ensure that it was at least available during normal clinic opening hours.

There was no requirement to access the National Poisons Advisory Service during the Games.

\section{EXPERIENCE AND EXPERTISE GAINED THROUGH THE GAMES}

It is patently clear that the members of the LOCOG PPC have, during the 4-year period of planning the Games, expanded their professional and personal knowledge and skills in multiple directions. It is also evident that the team of pharmacy Games Makers has benefited from the training programme provided prior to the Games and through their close association with athletes, healthcare professionals and other persons with whom they have had the privilege of interacting during the Games.

\section{KEY LESSONS FOR FUTURE GAMES}

In our opinion, the pharmacy services at the London 2012 Olympic and Paralympic Games were among the most advanced and comprehensive of any Olympic Games. However, the PPC have learnt valuable lessons from their experiences which will be relayed back to the International Olympic Committee to ensure continued development and improvement of services at future Games.

\section{Key messages}

- Pharmacy clinical services are a key component of medical services for major sporting events such as Olympic Games.

- The elements of these services require extensive and careful planning prior to the event and expert delivery by well-trained professionals.

Contributors The authors of this paper all made a substantial contribution to the analysis and interpretation of data, drafting and revising the content and providing final approval of the version to be published.

Funding None.

Competing interests None.

Provenance and peer review Not commissioned; internally peer reviewed. 